\title{
Human Articulation Efforts Estimation in the Automobile Vehicle Accessibility Movement - A Pilot Study
}

\author{
Jean-François Debril ${ }^{1}$, Philippe Pudlo ${ }^{1}$, Mohand Ouidir Ait El Menceur ${ }^{1}$, \\ Philippe Gorce ${ }^{2}$, and François Xavier Lepoutre ${ }^{1}$ \\ ${ }^{1}$ LAMIH UMR CNRS 8530, \\ Université de Valenciennes et du Hainaut-Cambrésis, Le Mont Houy \\ 59313 Valenciennes Cedex 9, France \\ \{jean-francois.debril, philippe.pudlo, mohandouidir.aitelmenceur, \\ francois-xavier. lepoutre\} @univ-valenciennes.fr \\ ${ }^{2}$ HANDIBIO-ESP EA 31-62, \\ Université du Sud Toulon et du Var, Avenue de l'université, \\ 83957 La Garde, France \\ philippe.gorce@univ-tln.fr
}

\begin{abstract}
To build a criterion of discomfort evaluation, based on the kinematics and the dynamics of the performed movement, is an expectation of the car manufacturers. There is no reference concerning the evaluation of the articulation efforts during the automobile vehicle accessibility movement. On this point, we propose to give a first reference. Our method and our computation were verified by a well known movement, the walking. The automobile vehicle accessibility movement of a young and healthy subject is studied. The articulation efforts presented are coherent with the produced movement's analysis.
\end{abstract}

Keywords: Automobile vehicle accessibility, Inverse dynamics, Articulation efforts.

\section{Introduction}

To enter or leave an automobile vehicle is a complex action requiring a precise coordination of the articular movements of the human body [7]. Felt at the time of its realization is an important factor for the customers mainly if this latter are elderly or having locomotor apparatus dysfunctions [14]. Traditionally, the car manufacturers realise ergonomic evaluation of the ingress/egress movements on real scale prototypes or mock-ups. This one is carried out with a significant number of subjects representative of the targeted customers. The subjective judgement given by the people taking part in the experiments is then correlated with dimensions of the vehicle [16], [1]. These approaches imply times and costs of significant treatment. If this approach is of habit, another possibility emerges and aims at evaluating discomfort only from the produced movement [4]. To go in this direction, our objective is to build a criterion (or criteria) of discomfort evaluation based on kinematics (e.g. articular angles) and the dynamics (e.g. articular torques) of the performed movement. 
For the moment, only discomfort evaluation based on kinematics was approached [4]. Moreover, until now, the articular torques estimation of the automobile vehicle accessibility movement was never studied.

The objective of this paper aims at the calculation of the articulations' efforts developed in the lower limbs while performing vehicle accessibility movements. A first step is to validate our method with a well-known data of the literature, a walking data. The second step is to use our method for the accessibility movement and to verify its relevance with an ergonomic analysis.

In a first time we will present the methodology that we have followed in our study, where the biomechanical model and the mathematical tools used in the modelling will be detailed. The experiments allowing the determination of the different parameters of the model will be then presented. Some data processing will come after that. Then we will present the results. Finally our paper will be concluded and some perspectives will be presented as well.

\section{Methodology}

\subsection{Theoretical Postulates}

The body segments are considered solid and indeformable during the movement. For this reason, the positions of the centre of gravity, the mass and the moment of inertia in the centre of gravity are considered constant.

The articulations are considered perfect, without friction and slip. The movement generation is due only to the articulations' torques.

\subsection{Kinematic Model}

The vehicle accessibility movement is a three-dimensional movement [1]. A multichain/poly-articulated three-dimensional skeletal model is chosen [6].

The physiology of the human body breaks up the lower limb into 3 segments [8]: the thigh, the leg and the foot. These segments form a simple kinematic chain for each of the two lower limbs, right and left. These 2 kinematic chains are articulated on the pelvis, taken as body of reference, whose position and orientation are known at every moment.

The articulation of the different body segments is modelled by perfect connections of spherical type. The hip, the knee and ankle are defined by 3 DOF, this makes 9 DOF per chain or $18 \mathrm{DOF}$ for the whole model.

The position and the orientation of the different segments the ones in opposite to the others and to the referential of reference $\mathrm{R}_{0}$ are defined by homogeneous matrices

${ }_{i+1}^{\mathrm{i}} \mathrm{T}$ (1):

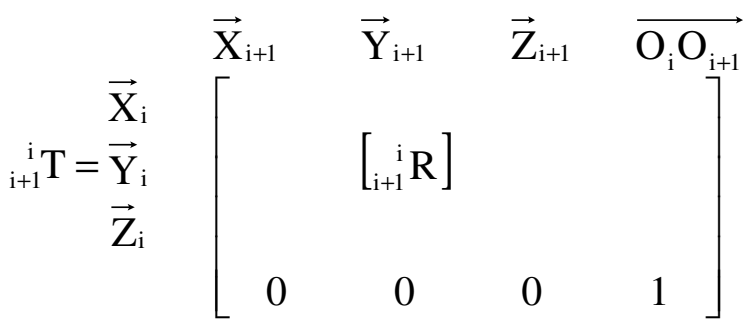




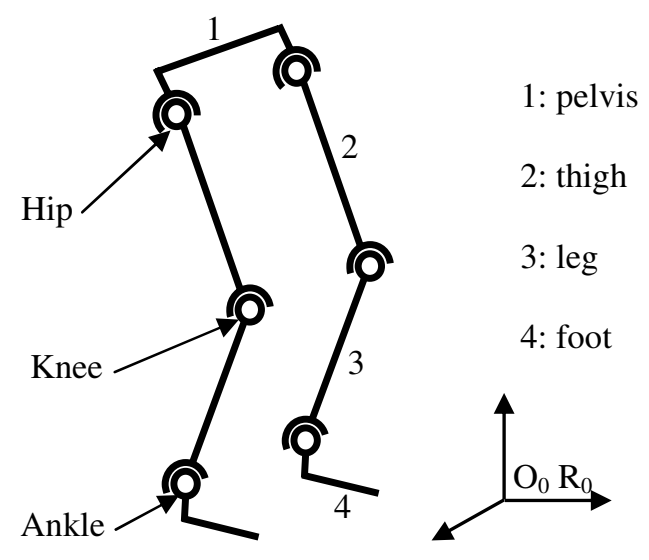

Fig. 1. The human body lower limbs' kinematic model

- $\overrightarrow{\mathrm{O}_{\mathrm{i}} \mathrm{O}_{\mathrm{i}+1}}$ represents the position of the medium of the pelvis or those of the articular centers into the referential $\mathrm{R}_{i}$.

- $\left[{ }_{i+1}^{i} R\right]$ represents the matrix of rotation of the referential $R_{i}\left(O_{i}, \vec{X}_{i}, \vec{Y}_{i}, \vec{Z}_{i}\right)$ in opposite to the referential $\mathrm{R}_{i+1}\left(\mathrm{O}_{\mathrm{i}+1}, \overrightarrow{\mathrm{X}}_{\mathrm{i}+1}, \overrightarrow{\mathrm{Y}}_{\mathrm{i}+1}, \overrightarrow{\mathrm{Z}}_{\mathrm{i}+1}\right)$.

The local referentials $R_{i}$ are built for each body segment according to the recommendations of the I.S.B. (International Society of Biomechanics). The matrix of rotation ${ }_{i+1}^{i} R$, ortho-normalized, is then obtained by the $z, x, y$ sequence Euler formalism [17].

The position of the end effector, the $2^{\text {nd }}$ metatarsal, in the referential related to the pelvis $R_{1}$ is expressed according to the equation (2):

$$
{ }_{4}^{1} \mathrm{~T}={ }_{2}^{1} \mathrm{~T} \times{ }_{3}^{2} \mathrm{~T} \times{ }_{4}^{3} \mathrm{~T}
$$

The position of the end effector in the referential of reference $\mathrm{R}_{0}$ becomes:

$$
{ }_{4}^{0} \mathrm{~T}={ }_{1}^{0} \mathrm{~T} \times{ }_{4}^{1} \mathrm{~T}
$$

\subsection{Angles Correction}

During the kinematics movement reconstruction, an error appears between the ankle calculate position and ankle measured position. This error is due amongst others to the instrumental errors and the experimental errors. This can generated a collision of the foot with the vehicle's sill during the automobile vehicle accessibility movement. The coherence of the kinematic reconstruction with the measured movement is 
evaluated using a calculation of correlation coefficient and RMS. If a consequent skew is noted, the ankle's position is optimized by a correction of the articular angles [12], [13].

\subsection{Anthropometric Model}

The building of the lower limbs' anthropometric model requires, for each body segment, (the foot, the leg and the thigh) to determine its mass, geometrical and inertial properties. For this study, the regression equations [18] are used to obtain, starting from a series of the studied subject's anthropometric measurements:

- The mass of the segments,

- The position of the centre of gravity along the longitudinal axis of the segments,

- Moments of inertia of the segments in the centre of gravity around their principal axes.

\subsection{Calculation of the Internal Efforts}

The estimation of the articulation efforts concerns an inverse dynamics problem. For this purpose, the Newton-Euler dynamic equilibrium expression is used.

The Newton-Euler dynamic equilibrium is written by using the formalism of the homogeneous matrices [10], [11]. It facilitates the writing of the recursive algorithm of inverse dynamics [3]. Moreover, this formalism makes it possible to follow the recommendations of the I.S.B. (International Society of Biomechanics) [17].

\section{Experiment}

\subsection{Device}

The experimental device is similar for the walking experimentation and for the accessibility experimentation. The experimental device aims the capture of the movements. Three types of data are desired: anthropometric, kinematic and dynamic.

The kinematics data are obtained by an optoelectronic motion capture system VICON®. The eight cameras are laid out around each vehicle. The system records the Cartesian position of the reflective markers laid out on the vehicle and on the subject.

Two KISTLER $®$ force plates are used to measure the ground reaction forces. The external efforts are applied at the end of the chain kinematics. Force plates are set in the medium of the way of walk or near to each vehicle in the case of ingress/egress movement.

Four vehicles of various dimensions, present in the trade, were used: a small car, a median car, a minivan and a small utilitarian. To allow the capture of the movements, the vehicles were stripped to the maximum. We present the small utilitarian's results.

\subsection{Protocol}

The base of the protocol is the same for the accessibility movement and for the walking movement. 
Anthropometric measurements carried out are those necessary to the anthropometric model [18].

Twenty markers, 16 anatomical and 4 technical, are laid out on each subject : $1^{\text {st }}$ and $5^{\text {th }}$ tarsals, medial and lateral malleolus, medial and lateral tibial condyles, anterior superior iliac spines, pubis and vertebra L5.

For the two applications, a capture of the lower limbs' circumduction movement is carried out. This one allows the determination of the articulation centres of the hips by a using the sphere fitting method [9], $[1<]$. The subject in sitting position is captured for the estimation of the marker L5 when it is masked by the automobile seat [5].

On the one hand, a walking movement is captured and on the other hand, four ingress/egress movements on each of the 4 vehicles are captured. The repetition aims to remedy the occlusion or the fall of reflective markers.

\subsection{Population}

Forty one subjects carried out the vehicle accessibility movement's protocol. The population is varied: young and healthy subjects, elderly subjects and disabled subjects. This study is interested on a young and healthy subject: 27 years old, $1.75 \mathrm{~m}$ and $74 \mathrm{~kg}$.

For the walking movement, the presented subject is also a young and healthy subject: 25 years old, $1.87 \mathrm{~m}$ and $80 \mathrm{~kg}$.

\section{Data Processing}

\subsection{Evaluation of Articular Kinematics}

Initially, the positions of the reflective markers measured by system VICON ${ }$ are filtered. A forth order Butterworth filter without lag at $5 \mathrm{~Hz}$ is used. In the second time, the parameters of the kinematic model are calculated.

Determination of the articular centers. The center of the knee is estimated at the median position of the markers laid out on the internal and external condyles and the centre of ankle at the median position of the markers laid out on the internal and external malleoli [2]. The position of the hips centres is determined by the calculation of sphere fitting [9], [15].

Determination of the medium of the pelvis and segmentary lengths. The segmentary lengths of the thigh and the leg are defined by the average distance between the hip and the knee on the one hand and between the knee and ankle on the other hand. In the same way, the width of the pelvis is defined as the distance between the two articular centers of the hips. The medium of the pelvis is defined in the medium of this segment.

Evaluation of the angular data. If need be, an optimization of the foot's position by correction of the articular angles is carried out. The corrected angles are considered 
valid when the movement reconstruction presents no collision of the foot with the vehicle's floor.

\subsection{Calculation of the External Efforts}

The torques of the mechanical action issued from the force plates are expressed in the local referential $\mathrm{R}_{\mathrm{k}}$. This information being disturbed, a filtering of the components of these torques is carried out by a forth order Butterworth filter at $5 \mathrm{~Hz}$.

The transfer of these torques in the referential of reference $R_{0}(10)$, makes it possible to meet the needs of the inverse dynamics algorithm.

$$
[\Phi]_{\mathrm{R}_{0}}={ }_{\mathrm{k}}^{0} \mathrm{~T} \cdot[\Phi]_{\mathrm{R}_{\mathrm{k}}} \cdot\left(\begin{array}{l}
0 \\
\mathrm{k}
\end{array}\right)^{-1}
$$

- $[\Phi]_{R_{k}}$ represents the filtered torque issued from the force plate k expressed in $R_{k}$,

- $[\Phi]_{\mathrm{R}_{0}}$ represents the filtered torque issued from the force plate $\mathrm{k}$ expressed in $\mathrm{R}_{0}$,

- ${ }_{\mathrm{k}}^{0} \mathrm{~T}$ represents the matrix of passage from the referential $\mathrm{R}_{\mathrm{k}}$ to the referential $\mathrm{R}_{0}$, and $\left({ }_{\mathrm{k}}^{0} \mathrm{~T}\right)^{-1}$ its reverse.

\section{Results}

\subsection{Walking Movement}

\section{Kinematics}

The reconstruction of the walking movement has a good fidelity with the trajectory of the feet:

- All the correlation coefficients between the positions of ankle measured and reconstructed are at 1.00. $(>0.95)$

- The RMS is $0.42 \mathrm{~cm}$. $(<1 \mathrm{~cm})$

This kinematics is used for the calculation of the articular efforts.

\section{Articular Efforts}

The results of this walking study seem to consolidate the experimental method, the kinematic model and the inverse dynamics algorithm (Fig. 2).

\subsection{Automobile Vehicle Accessibility Movement}

\section{Kinematics}

The reconstruction of ingress-egress movement presents difference between the trajectory of feet built and reconstructed (the RMS is $1.42 \mathrm{~cm}$ ). So the articular angles are corrected. Following optimization, the RMS is $0.67 \mathrm{~cm}(<1 \mathrm{~cm})$ and the correlation coefficients are at $1.00(>0.95)$. This acceptable movement reconstruction is used for the calculation of the articular efforts. 

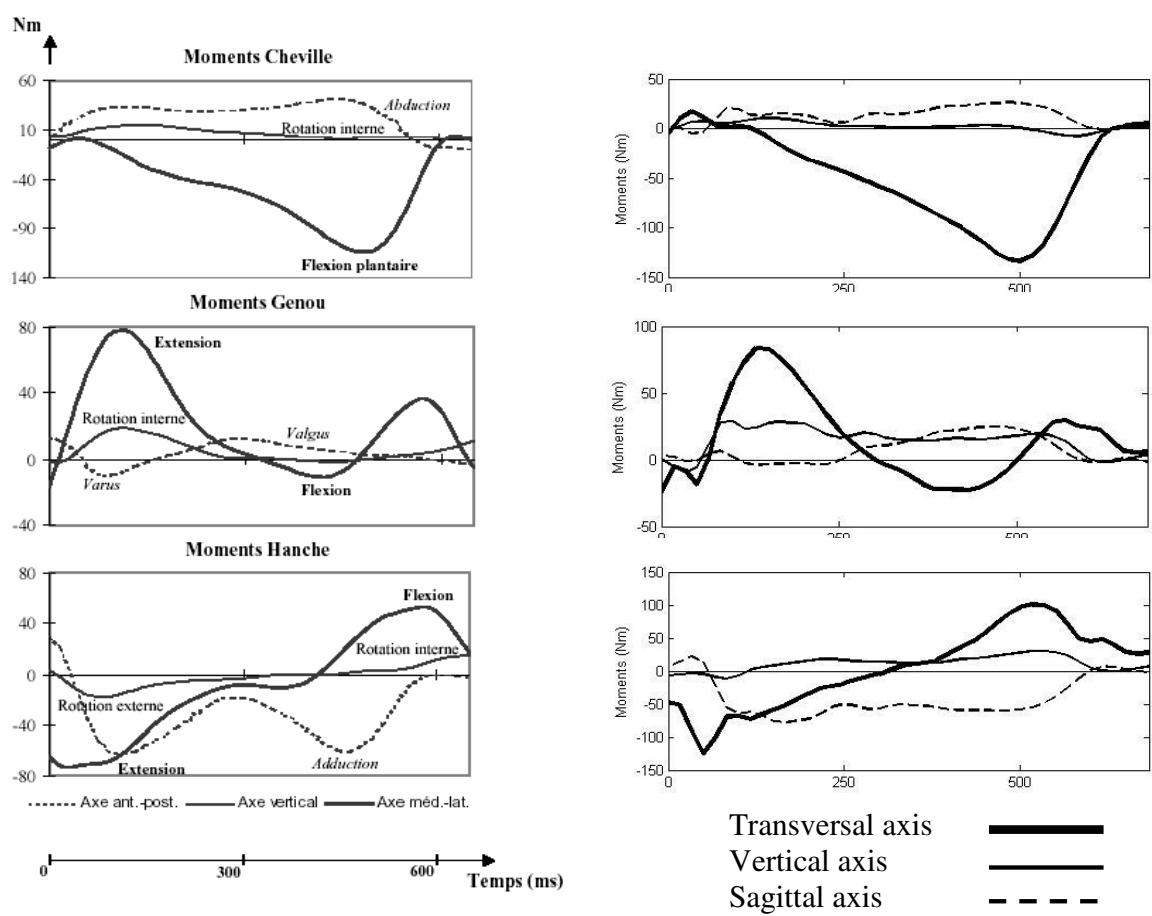

Fig. 2. Moments around the ankle (in top), the knee (in the medium) and the hip (in bottom), for the literature ([3] on the left) and for our subject (on the right) during the walking single stance

\section{Abduction-adduction efforts of the left hip during the ingress phase}

The figure 3 presents the comparison of the couples calculated around the sagittal axis and the articular angles associated. These results are at the end of the chain recursive algorithm.

\section{Analysis of the three phases}

Three phases are defined by the curves analysis (Fig. 3)

- A - concentric abduction phase

- B - concentric adduction phase

- $\mathbf{C}$ - excentric adduction phase

This analysis of the articular torques and kinematics corresponds to the ergonomic analysis. Phase A corresponds to the concentric opening of two lower members, which allows the subject to pose its right foot inside the vehicle. From this support, the phase $\mathbf{B}$ starts. The subject relocates its buttocks on the seat by tightening its legs. This is a concentric movement. Phase $\mathbf{C}$, the subject begins to get down towards the seat. It is a light eccentric opening that it retains its fall. 


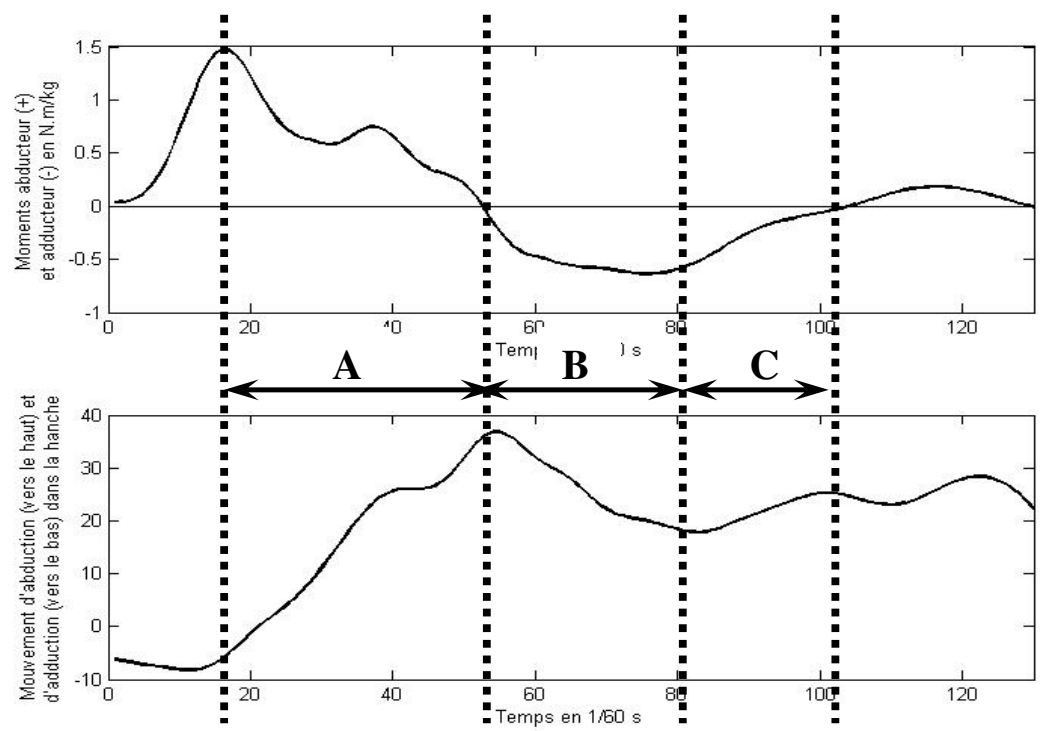

Fig. 3. Normalized torques by the weight and articular angles of abduction/adduction for the left hip during the phase of support unipode of entry in the small utilitarian (healthy subject of $1,75 \mathrm{~m}$ and $74 \mathrm{~kg}$ ). A: Takeoff of the right foot to its installation on the vehicle's floor. B: Placement of the buttocks to the top of the seat. C: Movement of descent towards the seat.

\section{Conclusions and Perspectives}

Kinematics model and anthropometric model were proposed. The articular efforts were estimated by inverse dynamics for the walking manipulation. The calculated articular torques are in coherence with the literature.

The automobile vehicle accessibility movement reconstruction require an optimization of the foot's position by a correction of the articular angles. The analysis of the results obtained by the calculation of dynamics is concordant with ergonomic analysis.

Moreover, for the automobile vehicle ingress movement, around the sagittal axis of the left hip, three phases were recognized: a concentric abduction phase since the takeoff of the right foot to its installation on the vehicle's floor, a concentric adduction phase corresponding to the transfer of the buttocks inside the vehicle above the seat, an eccentric adduction phase slowing down the fall of the subject during the descent in the seat.

The articular efforts are calculated for the lower limbs' articulations. The results obtained are coherent with the produced movement. The instrumentation being limited, the articular efforts can be calculated only for the beginning of the movement. More pushed instrumentation of the vehicle would allow an increased knowledge of the articular efforts.

A forthcoming study is interested in the population as a whole (elderly, disabled). The various assumptions of modeling and calculation are discussed there. 
Acknowledgments. Acknowledgments to the Ministry Delegated to the Higher Education and Research and to the IRRH and the IFRH for their support.

\section{References}

1. Andreoni, G., Rabuffetti, M.: New approaches to car ergonomics evaluation oriented to virtual prototyping. EURO-BME Course on Methods and Technologies for the Study of Human Activity and Behaviour, March 19-20 1997, Milano, Italy, pp. 1-13 (1997)

2. Cappozzo, A., Catani, F., Croce, U., Leardini, A.: Position and orientation in space of bones during movements: anatomical frame definition and determination. Clinical Biomechanics 10(4), 171-178 (1995)

3. Doriot, N.: Modélisation dynamique du membre inférieur pour l'estimation des forces articulaires et musculaires mises en jeu pendant la phase d'appui de la marche. $\mathrm{PhD}$ thesis, Université Claude Bernard, Lyon 1. N²59-2001

4. Dufour, F., Wang, X.: Discomfort assessment of car ingress/egress motions using the concept of neutral movement. In: SAE Digital Human Modeling For Design and Engineering Conference, Iowa City, Iowa, USA (June 15-17, 2005)

5. Gillet, C.: Analyse biomécanique de la marche et proposition de classes de marcheurs application au portage de sacs à dos. $\mathrm{PhD}$ Thesis. Université de Valenciennes et du Hainaut-Cambrésis (2004)

6. Gorce, P., Villard, C., Fontaine, J.G.: Grasping, coordination and optimal force distribution in multifingered mechanisms. Robotica 12(2), 243-251 (1994)

7. Gransitzki, K.: Biomechanics of entering a car. Second International Congress on Physiological Anthropology, University of Kiel, Germany (September 12-16, 1994)

8. Kapandji, I.A.: Physiologie articulaire, fascicule II, membre inférieur, Librairie Maloine S.A., Paris, ISBN: 2-224-01052-4

9. Leardini, A., Cappozo, A., Catani, F., Toksvig-Larsen, S., Petitto, A., Sforza, V., Cassanelli, G., Giannini, S.: Validation of a functional method for the estimation of hip joint centre location. Journal of biomechanics 32(1), 99-103 (1999)

10. Legnani, G., Casolo, F., Righttini, P., Zappa, B.: A homogeneous matrix approach to 3D kinematic and dynamics-I Theory. Mech. Mach. Theory 31(5), 573-587 (1996)

11. Legnani, G., Casolo, F., Righttini, P., Zappa, B.: A homogeneous matrix approach to 3D kinematic and dynamics-II Applications to chains of rigid bodies and serial manipulators. Mech. Mach. Theory 31(5), 589-605 (1996)

12. Lempereur, M.: Simulation du mouvement d'entrée dans un véhicule automobile. PHD thesis, Université de Valenciennes et de Hainaut Cambrésis. 06/04 (2006)

13. Lempereur, M., Pudlo, P., Gorce, P., Lepoutre, F.-X.: Optimization approach for the simulation of car accessibility movement. In: IEEE International Conference on Systems, Man and Cybernetics, Washington D-C, USA (October 5-8, 2003)

14. Petzäll, J.: The design of entrances of taxis for elderly and disabled passengers An experimental study. Applaid Ergonomics 26, 343-352 (1995)

15. Piazza, S.J., Okitaj, N., Cavanagh, P.R.: Accuracy of the functional method of hip joint center location: effects of limited motion and varied implementation. Journal of Biomechanics 34(7), 967-973 (2001)

16. Sternini, D., Cerrone, M.: Setup of an experimental procedure for measuring the human body movements when entering and exiting the vehicle. In: Proceedings of the 3rd International Conference on Vehicle Comfort and Ergonomics, pp. 257-265 (1995) 
17. Wu, G., Siegler, S., Allard, P., Kirtley, C., Leardini, A., Rosenbaum, D., Whittle, M., D'Lima, D.D., Cristofolini, L., Witte, H.: ISB recommendation on definitions of joint coordinate system of various joints for the reporting of human joint motion-part I: ankle, hip, and spine. Journal of Biomechanics 35(4), 543-548 (2002)

18. Zatsiorsky, V., Seluyanov, V.: In vivo body segment inertial parameters determination using a gamma scanner method, Biomechanics of Human movement: applications in rehabilitation, sports and ergonomics, Berme, N., Cappozo, A. (eds.): Bertec Corporation, Worthington, Ohio, U.S.A., pp. 196-202 (1990) 